ESJ Humanities

\title{
Dynamiques foncières et encastrement des marchés en Côte d'Ivoire : la fin du dogme du « Baoulé travailleur et non vendeur de terre $»$ ?
}

\author{
Dr. Mlan Konan Séverin
}

Enseignant-chercheur, Université Jean-Lorougnon Guédé-Daloa, Côte d'Ivoire

Doi: $10.19044 /$ esj.2021.v17n2p191

Submitted: 27 October 2019

Accepted: 30 December 2020

Published: 31 January 2021
Copyright 2021 Author(s)

Under Creative Commons BY-NC-ND

4.0 OPEN ACCESS

Cite As:

Mlan K.S. (2021). Dynamiques foncières et encastrement des marchés en Côte d'Ivoire : la fin du dogme du "Baoulé travailleur et non vendeur de terre »?. European Scientific Journal, ESJ, 17(2), 191. https://doi.org/10.19044/esj.2021.v17n2p191

\section{Résumé}

En Afrique, et singulièrement en Côte d'Ivoire, les peuples sont unanimes que la terre est un bien commun, sacré. Elle ne peut être cédée qu'à titre temporaire. En dépit de cette idéologie, il est apparu un marché foncier dans toutes les zones forestières et de transition. Cette étude part de l'hypothèse qu'une terre qui est favorable à une spéculation en " vogue » fait apparaître un marché foncier, qui subit les normes des autres marchés préexistants, et les normes traditionnelles de gestion foncière s'en trouvent déconstruites, dépassées. L'étude de type ethnographique a eu pour terrain empirique les villages des sous-préfectures de Kpouebo et de Kpacobo situés, respectivement dans les départements de Toumodi et Taabo (Centre-sud ivoirien), avec le village d'Adahou (dans la sous-préfecture de Kpouebo) comme cas de marché foncier. Les données ont été produites par une recherche documentaire couplée d'observations directes dans les villages desdites souspréfectures. De plus, des entretiens semi-directifs ont été faits auprès du Souspréfet de Kpouebo, du chef de village d'Adahou et sa notabilité, du président des jeunes et des membres de la mutuelle de développement d'Adahou, des chefs de ménages dudit village ayant accepté ou refusé de vendre leurs biens fonciers familiaux, enfin auprès des intermédiaires de vente de terre d'Adahou et de Kpouebo. Cette étude se fixe pour objectif d'appréhender tous les marchés fonciers comme des marchés encastrés, enchevêtrés dans des relations sociales locales et nationales. L'analyse des résultats montre que, tout 
comme les peuples forestiers qui n'ont pu s'abstenir de céder leurs terres, sous la ruée de migrants pour la cacaoculture ou l'urbanisation, les Baoulé du Centre-sud de la Côte d'Ivoire sont devenus «vendeurs » de terres rurales, sous la pression des élites politiques favorables à l'hévéaculture. Dès lors, l'apparition du marché foncier dans la zone d'étude doit être perçue comme les effets de l'encastrement des marchés fonciers d'autres régions forestières ivoiriennes, et non comme de la paresse caractérielle. En outre, il est à retenir que le marché foncier d'Adahou est spécifique, car la population a choisi de vendre des portions de terres familiales afin d'édifier un nouveau village et améliorer son cadre de vie. En somme, le marché foncier d'Adahou a engendré un nouveau village moderne. Cependant, ce marché n'a permis à la population de se créer des vergers d'hévéa. Il a plutôt entrainé le transfert de manteaux forestiers arables au profit des élites, rendant les propriétaires coutumiers des paysans sans terre.

Mots-clés : Dynamiques foncières, vente de terre, marché foncier, encastrement, Baoulé, Côte d'Ivoire

\title{
Land Dynamics and Market Building in Côte d'Ivoire: The End of the Dogma of "Worker Bulk and not Seller of Land"
}

\author{
Dr. Mlan Konan Séverin \\ Enseignant-chercheur, Université Jean-Lorougnon Guédé-Daloa, \\ Côte d'Ivoire
}

\begin{abstract}
In Africa and particularly in Ivory Coast, people are of the view that land is a common and sacred property. It can only be transferred temporarily. Despite this ideology, there is a land market in all the forest or transitional areas. This study starts from the hypothesis that a land which is favorable to a speculation in "vogue" gives rise to a land market, which is subject to the norms of other pre-existing markets, and the traditional norms of land management are thereby deconstructed and exceded. The ethnographic study took place in empirical land in the villages of Kpouebo and Kpacobo located respectively in the department of Toumodi and Taabo (in the middle-south of Ivory Coast) with the village of Adahou (in the sub region of Kpouebo) as an example of land market. The data was produced by a documentary research coupled with direct observations in the villages of the said sub-prefectures. Moreover, semi-structured interviews have been carried out with the sub prefect of Kpouebo, the chief of the village of Adahou and its leading members, the youth representative and the members of the association for the
\end{abstract}


development of the village of Adahou, the Chief of the family of the village who have accepted or refused to sell their family lands, and finally with middlemen who use to sell Adahou and Kpouebo's land. This paper focuses on understanding all markets land as embedded one, entangled in local and national social relationships. The analysis of the results show that like other people living in forest who could not give up their lands under the pressure of migrants for cocoa or urbanization, the Baoule people located in the middle South of Ivory Coast are becoming the "sellers" of rural lands under the pressure of political leaders in favor of rubber. The emergence of the land market in the study area should be seen as the effects of the embedding of land markets in other Ivorian forest regions, and not as laziness. In addition, it should be remembered that the Adahou land market is specific, as the population has chosen to sell portions of family land in order to build a new village and improve its living environment. In short, the Adahu land market has spawned a new modern village. However, this market did not allow the population to create orchards of rubber trees. Rather, it resulted in the transfer of arable forest coats for the benefit of the elites, making the customary owners of the landless peasants.

Keywords: Lands dynamics, land selling, land market, embedding, Baoule, Ivory Coast

\section{Introduction}

Plus que les décennies passées, la question foncière prend davantage d'importance dans l'espace politique nationale et dans les stratégies locales de développement (Lavigne, 2002, 1998).

Le foncier représente «l'ensemble des règles, de représentations et des pratiques qui organisent la façon dont une société utilise et contrôle la terre et les ressources afférentes » (Le Bris et al. \& , 1991). En d'autres termes, c'est l'ensemble des relations qui se nouent entre les individus et la terre, ainsi que les ressources renouvelables dont la terre est le support. Pour Stamm (1998), le foncier peut se concevoir comme un «fait social total », constitué à la fois par la terre et par l'ensemble des relations entre les individus et les groupes sur l'appropriation et l'utilisation des ressources. Selon lui, le support, le sol, facteur principal de production s'incruste dans une forte dimension religieuse, culturelle et affective. En Afrique, la terre est une « divinité, une génitrice nourricière » qui favorise le développement des hommes (Kouassigan, 1966). Elle occupe une place importante chez les différents peuples. La terre est donc sacrée, et de ce fait inaliénable pour les peuples comme pour les ancêtres ; elle est l'objet d'une appropriation collective (Mlan, 2013 ; Blanc-Pamard, 1981). En Côte d'Ivoire, les peuples sont unanimes : la terre est un bien commun ; elle est indivise, et ne saurait être cédée ou vendue définitivement (Tarrouth 
\& Colin, 2016 ; Mlan, 2013 ; Dijon \& Ndongmo, 2011 ; Kouassigan, 1966). Selon Dijon et al. (2011) «Les traditions africaines savent ce qu'est le bien commun : la terre des ancêtres, l'hospitalité envers l'étranger, la solidarité du village ». En effet, chez les groupes ethno-linguistiques de la Côte d'Ivoire (Gur, Mandé, Krou Akan), la terre est sacrée, incessible (Le Meur, 2008). Sur cette base, la vente d'une portion de terre est donc perçue chez tous les peuples de la Côte d'Ivoire comme une transgression de la coutume, la cession d'un bien commun. Selon Copagen et al. (2015), dans la culture africaine, la terre constitue le ciment de la spiritualité des peuples. Elle est le gage de leur existence. Elle fait partie du patrimoine incessible et inaliénable des communautés. En in fine, la terre « est intrinsèquement liée à leurs modes de vie, à leurs pratiques séculaires et à leur histoire. Elle porte en elle la promesse de leur avenir. Ainsi, la terre ne se vend pas. La terre est sacrée » (op cit).

De ce fait, dans la dynamique des mutations sociétales, les peuples chez lesquels des spéculations ont suscité la vente de terre sont vus comme des «gens paresseux », c'est-à-dire n'ayant pas de culture, aimant trop l'argent, la facilité. En clair, dans la littérature sur le foncier rural en Côte d'Ivoire, le peuple Baoulé, qui qualifiait tous les peuples de l'Ouest de " paresseux », n'est précédé que d'éloges perçus comme suit: (i) relativement à sa force d'intégration et de réussite, comme le mentionne Ibo (1995), avec le cas de Yao Appela, qu'il présente comme le modèle de migrant Baoulé ayant connu la réussite dans la zone d'immigration de Sassandra; (ii) au regard de sa capacité à coloniser la forêt (Mlan, 2004 ; Lena 1979; Schwartz, 1979), (iii) relativement à sa dynamise cultural (Zamblé, 2019; Kouadio \& Aloko-Nguessan, 2015 ; Babo \& Droz, 2008 ; Koffi, 1991 ; Lesourd, 1988¹) ; et (iv) vu leur mobilisation généralisée pour une forte émigration dans la zone forestière ivoirienne (Lesourd, 1982 ; Etienne et al., 1968 ).

Cependant, depuis le milieu des années 2000, les élites politiques et les hauts cadres de l'administration publique et privée ont commencé à percevoir la terre comme le meilleur investissement, en raison de la hausse du prix du latex d'hévéa (Mlan, 2015a ; Ruf, 2013, 2012). Alors, les Baoulé de la zone de contact forêt-savane des sous-préfectures de Taabo, Kpacobo, Kpouebo sont devenus des vendeurs de terres rurales (Tarrouth \& Colin, 2016; Mlan, 2015a, 2015b). L'indexation du Baoulé vendeur de terre apparait alors comme un paradoxe, une nouvelle réalité. De fait, le marché foncier ivoirien est encastré : de l'Est à l'Ouest en passant par le Sud, tout comme dans le Centre (Sud du département de Toumodi), l'exemple d'un encastrement des acteurs et pratiques est plus que prégnant (Colin et al., 2017). Vu sous cet angle, l'adage suivant lequel «les Baoulé sont travailleurs, et ne vendent pas de

${ }^{1}$ Lesourd (1988), dans le résumé de son travail affirmera que « Le système de production baoulé dans les zones de colonisation agricole de Côte d'ivoire forestière, et particulièrement du Sud-Ouest, est remarquablement efficace ». 
terre » tient difficilement, au regard de la naissance d'un marché foncier chez les Baoulé, au Sud de Toumodi.

L'hypothèse, c'est que, quand une terre ou une zone est favorable à une spéculation en "vogue », au plan national comme international, alors le marché qui apparait, semble-t-il presque spontanément, subit les normes des autres marchés préexistants ailleurs, et les normes traditionnelles de gestion foncière s'en trouvent déconstruites, dépassées. Tel est l'exemple des nouveaux marchés fonciers des années 2000 dans le Sud du «V Baoulé », notamment dans les sous-préfectures de Kpacobo et Kpouebo et département de Taabo.

L'objectif de cette étude est d'appréhender tous les marchés fonciers comme des marchés encastrés, enchevêtrés dans des relations sociales locales et nationales.

L'étude part de la thèse de Le Velly (2002), pour qui, «tous les marchés sont encastrés. Les échanges marchands se réalisent dans un contexte enchevêtré de relations interpersonnelles, de règles formelles, d'outils et de représentations collectives ». Chez Le Velly, tous les peuples vivent dans un système global d'encastrement. Les pratiques marchandes dans une zone donnée ne peuvent pas être circonscrites que dans cette seule zone. Selon Le Velly, les conditions d'encastrement à la fois contraignent et engendrent en aval les transactions marchandes. Pour Le Velly, les conditions $\mathrm{d}^{\prime}$ encastrement ${ }^{2}$ « ne constituent donc pas seulement le décor normatif où est prise la décision mais le socle, les ressources mobilisées continuellement lors de l'action ».

Par ailleurs, si dans l'ensemble des marchés fonciers ivoiriens, les cédants des terres utilisent l'argument de besoins financiers pour faire face à des obsèques de parents, à la scolarisation de progénitures, etc. (Colin \& Kakou, 2009), au niveau du village d'Adahou-Ngban ou Adahou plus simplement (sous-préfecture de Kpouebo), la création d'un nouveau village avec un habitat moderne se présente comme le motif majeur avancé par les cédants de parcelles de terre.

La méthodologie a privilégié une démarche qualitative axée sur l'approche ethnographique. Les données ont été produites par une documentation numérique et physique (au Centre de documentation de l'Institut de Recherche pour le Développement-IRD à Abidjan) mais également par des observations directes à Adahou, dans les villages des souspréfectures de Kpouebo et Kpacobo, appartenant respectivement aux départements de Toumodi et de Taabo, en Mars 2017 et en Décembre 2018.

${ }^{2}$ Karl Polanyi, Granovetter, Swedberd, et bien d'autres « ont en commun de considérer que l'économie n'est pas indépendante du reste de la société mais que, justement, l'économique est encastré dans le social » (Le Velly, 2002). 
En plus, 67 entretiens semi-directifs (par des guides d'entretien) ont eu lieu auprès du Sous-préfet et Chef du village de Kpouebo, du Chef du village d'Adahou et sa notabilité (10 membres), des chefs de ménages (gestionnaires de terres familiales d'Adahou) dont 38 et 7, respectivement, ont accepté et refusé de vendre leurs biens fonciers familiaux, et de 5 intermédiaires ou démarcheurs de vente de terre d'Adahou et de Kpouebo. En plus, le président des jeunes et 3 membres de la mutuelle de développement du village d'Adahou ont été interrogés.

L'étude s'articule autour de trois (3) parties : la première décrit le contexte écologique pré-forestier dans les sous-préfectures de Kpouebo et de Kpacobo favorisant la mise en place d'un marché foncier dans le «V Baoulé ». La seconde invite à une anthropologie de la marchandisation de la terre, et la troisième présente un cas d'émergence d'un marché à l'achat/vente et l'édification du nouveau village d'Adahou.

\section{Contexte écologique pré-forestier dans les sous-préfectures de Kpouebo et de Kpacobo}

La zone d'étude contient le creux du «V Baoulé », couvert de forêt au Sud-est, Sud et Ouest et la savane dans l'échancrure (Centre) (Mlan, 2015a ; Peltre, 1977 ; Adjanohoun, 1964). Dans son ouvrage «Le "BAOULÉ" (Côte d'Ivoire centrale) héritage géomorphologique et paléoclimatique dans le tracé du contact forêt-savane », Peltre (1977) situe l'aspect significatif du «V Baoulé » :

"Les savanes préforestières de Côte d'ivoire centrale qui pénètrent profondément sur près de deux cents kilomètres dans le massif forestier guinéen jusqu'à une centaine de kilomètres de la côte [mer], constituent avec le couloir des savanes du Togo-Dahomey l'un des accidents majeurs dans le tracé du contact forêt- savane en Afrique de l'Ouest ».

Aussi le territoire de Kpouebo et de Kpacobo, dans le creux du «V Baoulé » contient-il de vastes manteaux de forêt qu'encercle une savane très boisée de ligneux. Cette zone est très humide, car baignant dans deux bassins fluviaux, adossés à la forêt : le bassin du fleuve Bandama à l'Ouest, et celui de son affluent le Nzi, au Sud-Est. A partir de ces deux fleuves, on a le commencement de la forêt dense du Sud, de l'Est et de l'Ouest ivoirien. Les images ci-dessous indiquent le «V Baoulé ». 
Cartes n' 1, 2 et 3: Présentation du «V Baoulé » dans le Centre-sud de la Côte d'Ivoire
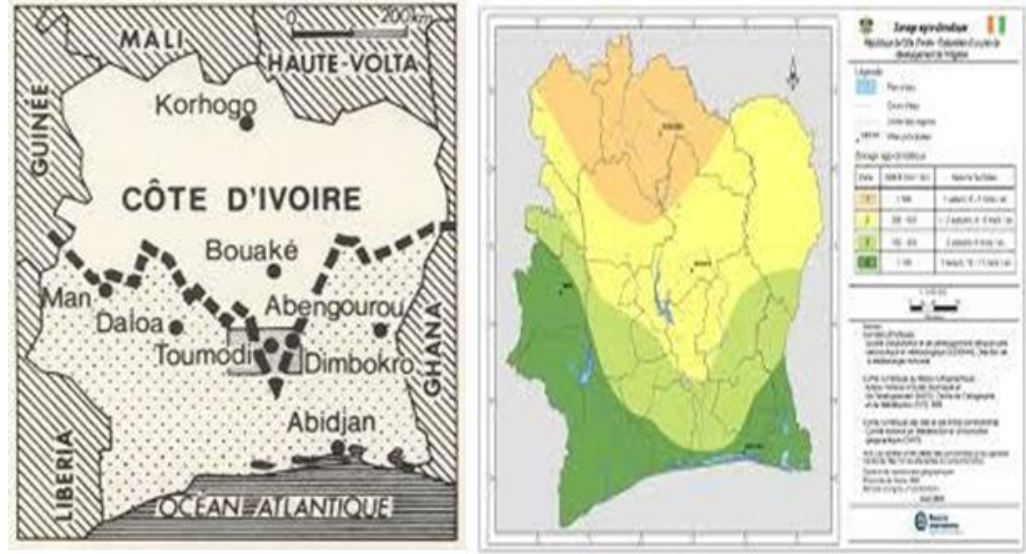

P Peltre

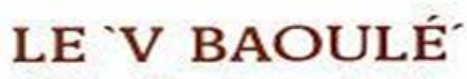

(Cote-d'Ivoire centrale)

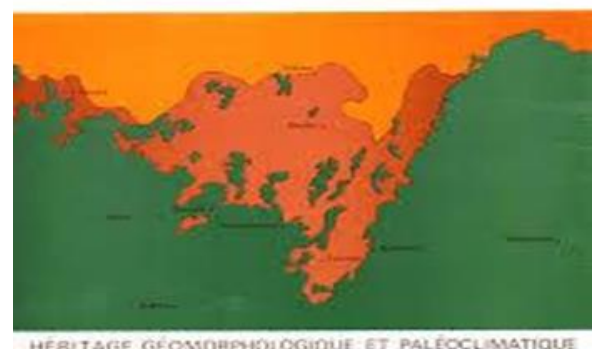

CANS LE TPGACE DU CONTACT FGAET-SMVANE

Source : Google, consulté le 10 Avril 2019

Selon Wikipedia (2019), ( Baoulé [bawle] est un peuple Akan et l'un des plus grands groupes en Côte d'Ivoire qui a migré du Ghana. Les Baoulé sont traditionnellement des agriculteurs qui vivent au Centre de la Côte d'Ivoire, dans une région en forme de triangle (le «V» Baoulé) entre les rivières Bandama et N'Zi ». Ancienne zone de production de café et de cacao, les sécheresses des années 1980 ont été à la base de deux phénomènes : il s'agit d'une forte émigration vers les zones forestières de Soubré, Duékoué, Guiglo, et d'une reconversion de la population résidente en producteur de riz pluvial. Jusqu'au début des années 2000, la sous-préfecture de Kpouebo étaient connue comme le grenier du bon riz paddy et autres cultures saisonnières des départements de Toumodi et de Taabo.

L'attrait du boom de l'hévéa (atteignant 1000 francs le kilogramme de latex en 2009-2010) (Ruf, 2012), à la même période, crée chez les cadres et élites (politiques et économiques) un effet d'encastrement du marché foncier : les tentatives de grands investissements pour cette spéculation se heurtant à la 
raréfaction foncière, les cadres et élites se tournent vers les zones de transition où la disponibilité foncière incite à la convoitise. A preuve, en 2007, un dirigeant politique ayant pour nom Z. K, originaire de Soubré (Sud-ouest), promoteur de grands investissements dans l'hévéaculture dans cette zone, obtient environ 1000 hectares à Dida-kouadiokro, à l'Est de Kpouebo (Mlan, 2015a). Son acte forge alors l'apparition d'un marché foncier en pays Baoulé (Colin \& Tarrouth, 2016 ; Tarrouth., 2016 ; Mlan, 2015a). Cela appelle une anthropologie politique de la marchandisation de la terre sur les Baoulé du Centre ivoirien.

\section{Anthropologie politique du « travail et vente » de la terre dans le «V Baoulé »}

\section{1 " Le Baoulé ne vend pas la terre ?"}

Selon l'adage ivoirien, le «peuple Baoulé est grand travailleur». Le principal facteur du développement prodigieux de la cacaoculture en Côte d'Ivoire est son adoption par les Baoulé. Ceux-ci représentent plus de $23 \%$ de la population nationale (Etienne et al ., 1971). Selon Adjanohoun (1964), « Le territoire de peuplement originel des Baoulé couvre $35000 \mathrm{~km}^{2}$ (de Katiola à N'douci, autour du 6e degré de latitude nord). Evalués environ à 800000 habitants à la fin des années 1960 (Etienne et al., 1971), les Baoulé entament alors une migration économique dite « de colonisation » de la forêt de l'Ouest depuis les années 1950 (Lesourd, 1988). Ainsi, le Recensement Général de la Population et de l'Habitat de la Côte d'Ivoire de 1975 fixe la population Baoulé à 1302055 habitants, soit 18,7\% de la population nationale (Lesourd, 1985), dont les immigrés sont dispersés dans plus de 50 villes du territoire national ». Ils se distinguent particulièrement par un dynamisme pour la migration (Viti, 1991 ; Lesourd, 1982).

Depuis les années 40, période de la création de villages Baoulé dans le Sud-est (Nzikro, Koffikro, Bénianklé, etc), ce peuple se présente comme le bras séculier de la politique de mise en valeur des terres prônées par le colon, devenue «slogan-loi » dans les années 1960 avec le Président Houphouët Boigny (Verdeaux, 2011).

En outre, au milieu des années 80, les Baoulé sont vus comme de gros travailleurs, en raison de leur capacité à occuper la forêt, et à créer de grandes plantations de cacaoyers (Koffi , 1991 ; Schwartz, 1979; Lesourd, 1982). Cette «colonisation agricole » est facilitée par une main-d'œuvre disponible dans les zones d'émigration (au sein de laquelle, on trouve une forte population voltaïque ou Burkinabé actuellement). Dans cette dynamique de travail, les aides-familiaux travaillent comme manœuvre, et par la suite, ils acquièrent leurs propres parcelles, et créent leurs propres vergers (Kouakou \& Mlan, 2019). 
Ainsi, sur la base des entretiens, relativement à leur capacité à «anthropiser » la forêt, et à la cultiver, les Baoulé considèrent les peuples d'accueil (Gban, Bété, Gouro, Bakwé, Kroumen, Neyo, Dida, Guéré, Wobé, Yacouba, etc.) comme des paresseux, ceux qui ne connaissent pas la valeur de la forêt. Mieux, ils s'appuient sur le marché foncier desdits peuples, qui a pu leur faciliter l'accès aux terres de forêts, pour affirmer qu'ils ne peuvent pas vendre les terres dans leurs zones d'origine. Pour eux, les peuples d'accueil allient « paresse » à l'esprit mercantiliste, faisant d'eux des irresponsables, des vendeurs à tout vent de terre, au regard des données empiriques.

Cependant, lorsque Z. K., le cadre politique de Soubré est arrivé à Dida-kouadiokro avec son «double jeux de pouvoir » politique et financier (Mlan, 2015c), les Baoulé ont fait fondre le dogme faisant d'eux des peuples travailleurs qui ne peuvent pas vendre leur terre.

Aussi dès 2008, la ruée des élites politiques et cadres dans la sous-préfecture de Kpouebo donne-t-elle lieu à un véritable marché foncier, avec incomplétude selon Colin et Tarrouth (2016), dans une rare compétition d'acteurs (demandeurs de terre, démarcheurs ruraux, propriétaires fonciers coutumiers), autour de l'achat/vente. Egalement, l'on observe que l'apparition d'un marché foncier est plus l'apanage de spéculation sur la terre d'une zone donnée que des « castes à bravoure » ou une quelconque tradition s'offusquant et s'opposant depuis originellement aux cessions de terre.

A priori, dans l'intersection du fleuve Ban dama et son principal affluent Nzi, plus forestière, là où le café, le cacao et l'hévéa trouvent des terreaux fertiles, la terre s'échange avec du numéraire, dans un contexte d'antagonismes et de conflits violents entre villages, avec affaiblissement des instances de règlement des litiges fonciers (Tarrouth \& Colin, 2016; Mlan, 2015a, 2015c). Quelle est la particularité du marché foncier dans le Centresud Baoulé ?

\subsection{Spécificité du marché foncier dans le «V Baoulé »}

De 2007 à 2018, le marché foncier dans les sous-préfectures de Kpouebo et de Kpacobo n'a donné lieu qu'à des vergers d'hévéa, appartenant tous aux élites politiques et cadres ${ }^{3}$. A fortiori, les Baoulé travailleurs, autochtones, sont également devenus " paresseux », oisifs. Les démarcheurs ruraux, pierre angulaire de ce marché, également Baoulé, deviennent par la suite manœuvres des élites et cadres politiques sur les terres arables nouvellement acquises.

Qu'est-ce qui différencie le marché foncier du «V Baoulé » de celui de l'Ouest ivoirien ? Si chez l'ensemble des peuples de l'Ouest ivoirien (Bété, Dida, Neyo, Kroumen, Bakwé, Wê, Gouro, Yacouba, Gban, etc.), les résidents

\footnotetext{
${ }^{3}$ Selon Tarrouth (2016) : ils sont considérés comme des entrepreneurs ruraux.
} 
ont quelques vergers de cacaoyers, parsemés d'essences ligneuses (iroko, fromager, Spondia) et autres arbres fruitiers (oranger, manguier, avocatier, goyavier, mandarinier), chez les Baoulé de Kpouebo et Kpacobo, les résidents n'ont pu jusque-là cultiver le moindre verger d'hévéa. Pire, ayant vendu les terres arables leur servant de facteur pour la riziculture pluviale, les Baoulé de Kpouebo et de Kpacobo ne deviennent que de simples paysans sans terres. C'est le cas de N. K. qui l'exprime en ces termes :

"Je suis démarcheur si quelqu'un veut payer ou vendre la terre. Mon grandfrère et moi avons vendu 36 hectares de la famille à un $\mathrm{Mr}$ qui est grand cadre au port d'Abidjan. Après, on ne sait plus où cultiver. Je suis allé voir Yao B, mon preneur de terre, pour être manœuvre dans sa plantation».

Si sur le marché foncier de l'Ouest de la Côte d'Ivoire les cédants de terre s'accrochent à leur « caste » de tuteur, attendant une assistance sociale (aide financière en cas de difficultés, habits, huile, etc.), en raison des cessions de terre qui n'étaient parfaites comme l'entrevoient l'essentiel des écrits sur les transactions foncières en Côte d'Ivoire (Tarrouth \& Colin, 2016), chez les Baoulé de Kpouebo et de Kpacobo, les élites et cadres politiques, accueillis parce que considérés comme partenaires, se détournent de ces valeurs sociales ivoiriennes. Ces « partenaires » n'assistent ni leurs tuteurs ni les villages. Ces cédants-Baoulé ne se contentent que de petits champs d'igname, de banane sur des sols de plus en plus pauvres. A la suite de la présentation du marché foncier $\mathrm{du}$ «V Baoulé », le marché de la terre du village d'Adahou se présente comme un exemple.

\section{Emergence d'un marché à l'achat/vente et édification du nouveau village d'Adahou}

\subsection{Idée de marchander la terre, le « bien commun divin »}

Adahou est situé à l'Ouest de Kpouebo, son chef-lieu de souspréfecture (département de Toumodi). Adahou est situé sur l'axe MoronouAkakro-Kalékoi à 12 km de Moronou au Nord-ouest, à 06 km de Kpouèbo au Nord-Est et à $13 \mathrm{~km}$ de Kalékoi au Sud. Adahou compte 14 campements, peuplés essentiellement d'allochtones Baoulé (venus d'autres régions Baoulé) et de Burkinabé.

Or, la terre est un support sacré, divin, chez les Baoulé, de même que chez les Baoulé Ngban d'Adahou (Blanc-pamard, 1981). Pour N'Gouamidou (2000), la terre revêt d'un caractère très capital dans les sociétés rurales africaines. Les différentes croyances africaines présentent la terre comme un bien hautement sacré. C'est le trait d'union entre le monde des vivants et celui des morts selon certaines croyances. Ainsi perçue, la terre acquiert-elle un droit inaliénable, et est considérée comme un bien collectif. Pour les africains, la terre est une « divinité, une génitrice nourricière » qui répond aux besoins 
des hommes (Kouassigan, 1966). Elle occupe une place importante dans les sociétés, car étant leur principal moyen de production et leur première source de revenu. Cette ressource foncière est de ce fait inaliénable pour ceux-ci, car appartenant aux ancêtres et à la progéniture d'où son appropriation collective, dans les relations de parenté qui représentent une institution centrale (Colin, 2004).

Aussi la mise en marché de la terre à Adahou pour le développement d'un nouveau village sur un autre site a-t-elle été le fruit de la mutuelle «EBOHEKOUN », qui signifie « unissons-nous » regroupant des cadres, travailleurs, élèves et étudiants d'Adahou. Selon le chef de ce village, la motivation de la création de cette mutuelle est la suivante: "nous avons mis en place une mutuelle pour développer notre village, et cette association, on l'appelle 'EBOHEKOUN". Y'a une caisse pour ça où chacun doit cotiser chaque fin du mois trois mille franc CFA ». En effet, tout a commencé vers les années 2000. Lors d'une réunion, les membres de la mutuelle ont fait l'amer constat que l'ancien village est encerclé par le cimetière, et que le sol est détruit par l'érosion, avec des cases accolées dont certaines sont presqu'en inclinaison, offrant le risque d'écroulement. Comme solution, le lotissement d'un nouveau site pour le nouveau village a été décidé.

Mais, les populations devraient faire face à la mobilisation du facteur financier. Alors, lorsque les élites politiques et économiques ont commencé à sillonner la zone, à partir de Dida-yaokro et Dida-kouadiokro, la chefferie du village et la mutuelle ont convenu que chaque famille vende une partie de son patrimoine foncier, en vue de l'édification d'un habitat moderne sur le nouveau site loti.

Comme prévu, et par les intermédiaires issus des villages de Kpouebo et d'Adahou, les propriétaires terriens seront sollicités, dès l'année 2008 par une ruée de ministres, militaires gradés, directeurs d'administrations centrales et privées, fonctionnaires de toutes catégories, etc. (Mlan, 2015c). Selon K. K. (Douanier du village d'Adahou), sans toujours recourir à tous les membres de chaque famille, des individus, désireux de bâtir leurs concessions ont cédé entre 100.000 et 120.000 francs CFA l'hectare de terre. Par 50 à plus de 1000 hectares en un seul tenant, sur les patrimoines de plusieurs familles, le marché foncier a dépassé les attentes de la population (Tarrouth, 2016 ; Mlan, 2015a).

Comment le chef du village d'Adahou perçoit le marché foncier en cours dans son village? Il ressort des entretiens les propos suivants: "Aujourd'hui, c'est grâce à ça qu'on a construit notre école primaire que tu vois là mais c'est le gouvernement qui nous envoie les enseignants; et puis on a fait venir deux pompes [HV] nous-mêmes d'abord et puis le gouvernement a donné un». 
Quels sont les matériaux et les types d'habitat que le marché foncier d'Adahou a suscité ? Ci-dessous, les images sont de l'habitat du nouveau village d'Adahou.

Photos 1 et 2 . Maisons modernes et semi-modernes

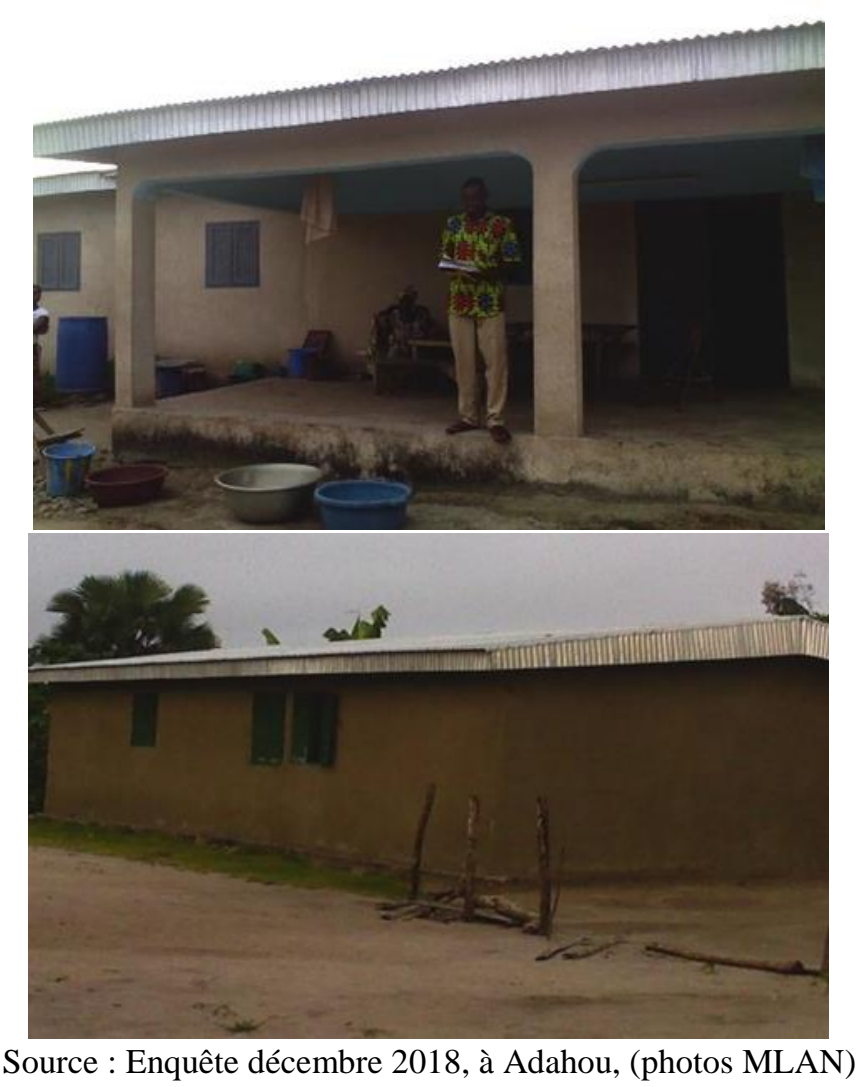

La marchandisation de la terre à Adahou a engendré trois types d'acteurs et d'habitat :

- la construction de maisons en dur (briques, fer, avivés d'iroko, sciés le plus souvent sur place, etc.) est le fait de personnes qui ont vendu l'essentiel des patrimoines familiaux et lignagers;

- l'édification de maisons qualifiées de semi-modernes, comportant un mur en banco crépi et la tôle à la toiture, par l'utilisation d'avivés (planches) de tronc sec de rônier (voir photo 1 et 2), est initiée par des cadres moyens, des paysans migrants ou résidents qui ont vendu des superficies pas trop grandes (entre 10 à 25 hectares) ;

- la construction de cases (avec un mur en banco crépi ou non, et la toiture en paille) qui est faite par les personnes ayant vendu la terre, en superficie moindre (moins de 10 ha) ou ayant refusé de céder ou «brader » leurs biens fonciers. 
L'existence de ces maisons modernes justifie la vente des terres à Adahou. Elles ont permis de transformer le rêve d'édification de concessions modernes en réalité, grâce à la vente de la terre. Le tableau ci-dessous présente la proportion des maisons dans ce nouveau village.

Tableau 1 . Niveau de modernisation de l'habitat d'Adahou par le fait du marché foncier.

\begin{tabular}{|l|l|l|l|l|}
\hline Type de maison & $\begin{array}{l}\text { Maisons } \\
\text { traditionnelles }\end{array}$ & $\begin{array}{l}\text { Maisons semi- } \\
\text { modernes }\end{array}$ & $\begin{array}{l}\text { Maisons } \\
\text { modernes }\end{array}$ & Total \\
\hline Nombre & 40 & 105 & 95 & 240 \\
\hline Pourcentage & $17 \%$ & $44 \%$ & $40 \%$ & $100 \%$ \\
\hline
\end{tabular}

Source : Données d'enquête, Décembre 2018 (MLAN)

Au regard du tableau, l'on note que les maisons modernes et semimodernes sont beaucoup plus présents par rapport aux maisons en case. Elles représentent respectivement $44 \%$ et $40 \%$ tandis que les cases sont estimées à $16 \%$. Cela traduit en quelque sorte la généralisation de la marchandisation de la terre. En plus de la modernisation des bâtisses, d'autres équipements sont réalisés à l'initiative de la mutuelle du village. L'image ci-dessous, montre l'école primaire publique et une pompe à motricité humaine au sein de celleci.

Photo 3 . Ecole primaire avec une pompe à motricité humaine sur le site du nouveau village d'Adahou

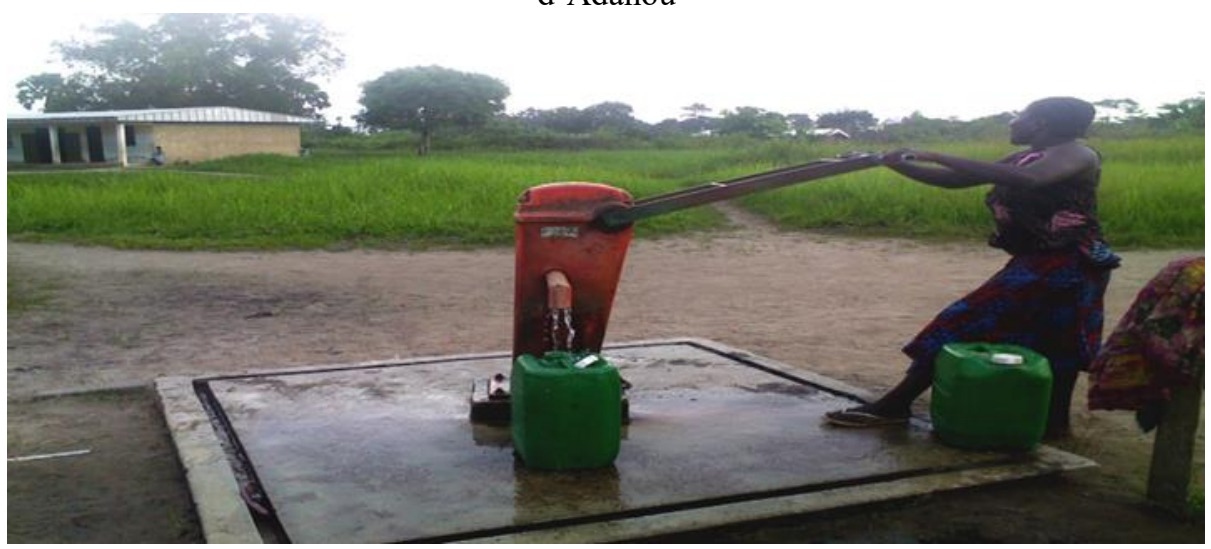

Source : Enquête décembre 2018, Adahou, (Photo MLAN)

Et alors, quels problèmes soulève l'émergence du marché foncier du village d'Adahou?

\subsection{Situation des terres rurales suite au marché foncier}

Selon les enquêtes de terrain, trois situations sont implacables sur le territoire villageois d'Adahou. La première, c'est l'épuisement total des réserves foncières familiales. Pour le Sous-préfet de Kpouebo, "la terre à Adahou, c'est du vieux souvenir. Si quelqu'un veut faire un champ, peut-être, c'est dans les forêts sacrées, qui existent autour du village. C'est un 
désastre! ». Alors, le président des jeunes regrette presque le phénomène de la vente des terres, en ces termes: "alors qu'on se moquait des gens de l'Ouest de la Côte d'Ivoire, aujourd'hui, je ne sais pas où nous allons trouver de la terre pour faire les champs pour nourrir nos familles : tout a été vendu ».

Lors de l'étude, le Sous-préfet s'est finalement montré très alarmant. Selon lui, lorsque tous ceux qui ont acquis de la terre à Adahou la mettront en valeur, tout le monde sera «paysans sans terre».

La deuxième concerne l'apparition de conflits fonciers sur toutes les cessions de terre (Mlan, 2015a). Les ventes de terre étant presque cachées, l'essentiel des membres des familles n'a pas été associé lors des contrats. Lorsque les gens sont informés de la cession de tous leurs patrimoines fonciers, le geste qui suit, c'est la plainte à la sous-préfecture de Kpouebo ou à la gendarmerie de Toumodi. Les propos K. K (capitaine de la Douane, fils d'Adahou) confirme cette situation: "j'ai appris qu'un ancien premier ministre a payé ma terre. Le jour où il va entamer la demande de titres fonciers, il me trouvera sur son chemin. Je l'attends!».

Et la troisième situation révèle le Baoulé comme vendeur de la terre, comme chez les peuples forestiers (Sud, Sud-ouest, Ouest). Peut-être même que les Baoulé vendent beaucoup plus la terre que tout autre peuple. Des années 20 jusqu'à ce jour, on note toujours quelques réserves forestières familiales chez les Agni (Mbatto, Bongouanou, Abengourou, Agnibilékrou, Aboisso, notamment), les Avikam (Grand-Lahou), les Néyo (Fresco, Sassandra), les Dida (Divo, Iré, Lakota), les Bété (Gagno, Daloa, Issia), les Kroumen (San Pédro, Tabou), les Guéré (Duékoué, Guiglo, Toulepleu), les Yacouba (Man, Danané), etc. Chez ces peuples, des migrants ont pu obtenir des terres par la force de travail sur deux à trois ans, sans salaire, pour la production de cultures de subsistance à leurs tuteurs, avant que ces derniers leur concèdent des parcelles de forêt. Mais, dans le «V Baoulé », en l'espace de douze ans (depuis 2007), la zone de Taabo, Nzianouan, Singrobo, Kpacobo, Awakro, Moronou, et le reste de la sous-préfecture de Kpouebo ne contient que quelques interstices de savane, toutes les terres arables de forêt ayant été vendues.

\section{Conclusion}

Le paradigme « orthodoxe » ou standard est formel : en cas de pression sur une terre, la tenure foncière tend vers une individualisation. Apriori, on ne saurait dénier au peuple Baoulé sa capacité à anthropiser la terre et à la mettre en culture. L'essor de la cacaoculture tient en grande partie de la bravoure de ce peuple.

Cependant, au regard de la dynamique du marché foncier dans le Sud du département de Toumodi, en pays Baoulé, il serait plus approprié de parler 
de «mauvais » jugement sur les peuples que les Baoulé considéraient comme paresseux et vendeurs de terre.

A fortiori, lorsque la terre d'une zone donnée se trouve favorable à une spéculation, le peuple concerné par cette zone subira, le double «jeux de pouvoirs politique et financier », inéluctablement. Ce n'est donc pas parce que certains peuples sont paresseux. Les demandeurs de terre utilisent des atouts asymétriques redoutables :

- La force de travail, au service du propriétaire foncier, rendant ce dernier dépendant, mais également reconnaissant (certains donnent leurs filles afin que cette force de travail reste et enrichisse la famille par la création de vergers de la part du migrant);

- Le double «jeux de pouvoirs politique et financier» hypnotise, déconstruit et désarme les propriétaires coutumiers, en ce que l'homme politique mobilise son influence sur les cadres, transformant ces derniers en guides, démarcheurs, partenaires, courtisant par endroit ; les moyens financiers façonnent puisque les populations rurales pensent qu'accepter un haut cadre, cela peut ouvrir des portes: équipements de base du village, insertion des diplômés, force de protection en cas de problème avec la justice...

Selon T.Z., anciennement patriarche Bakwé de Gnamagui (Soubré), l'accueil et l'installation de l'étranger, comme en parle Jacob chez les Gwendégué du Burkina Faso (2004), est un trait culturel africain ; ce n'est pas parce que les peuples ne connaissent pas la valeur des ressources naturelles dont ils en dépendent d'ailleurs. En in fine, les arguments qu'utilisent les populations, lors des cessions foncières, ne sont que de façade, puisqu'ils sont les mêmes partout. Oui, les Baoulé sont de grands travailleurs mais également peuvent être des vendeurs de terre si des spéculations les contraignent. L'absence de vergers d'hévéa chez les paysans autochtones du «V Baoulé » est la preuve qu'être agent de vente de terre rurale n'est pas le symbole de la «paresse »; c'est une mutation sociale.

En somme, même si le marché foncier d'Adahou a permis l'édification effective d'un nouveau village moderne, il est subséquemment à la base de la disparition des manteaux forestiers familiaux et de la riziculture, de l'apparition de paysans sans terre, de la paupérisation des paysans locaux. Le marché foncier n'a pas fait des Baoulé de la zone d'étude des planteurs d'hévéa. Si le Baoulé immigré est dynamique, ce n'est pas de même pour celui qui n'a pas connu de migration. En regard, les stéréotypes sur les peuples sont à proscrire pour une cohésion sociale. 


\section{References:}

1. Adjanohoun, E. (1964). Végétation des savanes et des rochers découverts en Côte d'Ivoire centrale. Paris, ORSTOM, (7), $178 \mathrm{p}$

2. Chauveau, J-P., Colin, J-Ph., Jacob, J-P., Lavigne Delville, Ph., \& Le Meur, P-Y. (2006). Modes d'accès à la terre, marchés fonciers, gouvernance et politiques foncières en Afrique de l'Ouest : résultats du projet de recherche Claims. IIED-GRET, $92 \mathrm{p}$

3. Babo, A. \& Droz, I. (2008). «Conflits fonciers. De l'ethnie à la nation. Rapports interethniques et «ivoirité » dans le sud-ouest de la Côte d'Ivoire ». In Colloque Les frontières de la question foncière : enchâssement social des droits et politiques publiques, IRD, 16 au 19 mai 2006 à Montpellier, pp741-764

4. Blanc- Pamard, C. (1981). Le foncier rural en Côte d'Ivoire, Ministère du plan et de l'industrie, Abidjan, 79p.

5. Colin, J-Ph . \& Kakou, A. (2009). Etude sur la location et les ventes de terre rurales en Côte d'Ivoire : rapport de la mission 2. Hemel Hempstead (GBR); Abidjan: HTSPE; Ministère de l'Agriculture, 138 $\mathrm{p}$

6. COPAGEN, Inter Pares, REDTAC (2015). Touche pas à ma terre, c'est ma vie! Abidjan, Inades formation

7. Dijon, X. \& Ndongmo, M. (Dir.) (2011). L'éthique du bien commun en Afrique. Regards croisés. L'Harmattan, Collection : Etudes Africaines, 212p

8. Etienne, P. \& Etienne, M. (1968). L'émigration Baoulé actuelle. Les Cahiers d'Outre-Mer, -21-82 pp. 155-195.

9. Ibo, J. G. (1995). Genèse de l'économie de plantation ivoirienne. Le cas de Yao Appéla, Cahiers d'études africaines /138-139/ pp 541-562

10. Jacob, J-P. (2004). Gouvernement de la nature et gouvernement des hommes dans le Gwendégué (centre-ouest du Burkina Faso, Autrepart, Armand Colin/IRD, pp 25-43

11. Kouassigan, G. A. (1966). L'Homme et la terre. Droits fonciers, coutumiers et droit de propriété en Afrique occidentale Paris, BergerLevrault, 283p, Bibliogr. (Coll. L'homme d'Outre-Mer).

12. Koffi, A. L. (1991). Les implications de la migration baoulé dans le sud-ouest ivoirien (San Pedro Soubré, Divo): une approche sociologique, Abidjan, ORSTOM

13. Kouadio, Y. D. \& Aloko-Nguessan, J. (2015). Dynamique démographique et économique, facteurs déterminants de la croissance spatiale des villes du département de Soubré (Côte d'Ivoire), European Scientific Journal, Edition vol.11, No.26 -1857 - 7881 pp 1980-199

14. Kouakou, Y. Fr. \& Mlan, K. S. (2019). Raréfaction foncière, absence de main d'œuvre et stratégies d'adaptation dans les activités agricoles 
à Boguedia (Issia Côte d'Ivoire). Revue Africaine de Criminologie $\mathrm{N}^{\circ}$ 25, Abidjan, pp211-221

15. Lesourd, M. (1988). La forêt, la machette et le billet de banque Problématique du colonat agricole en région d'économie de plantation : l'exemple du système de production Baoulé (Côte d'Ivoire forestière). Cah. Sci. Hum. 24 (1) pp 73-97.

16. Lavigne, D. Ph. (2002). Les pratiques populaires de recours à l'écrit dans les transactions foncières en Afrique rurale Eclairages sur des dynamiques d'innovation institutionnelle. IRD-GRET, Montpellier, $20 \mathrm{p}$.

17. Lavigne, D. Ph. (ed.) (1998). Quelles politiques foncières pour l'Afrique rurale? Karthala, Paris.

18. Le Bris, E., Le Roy, E., \& Mathieu, P. (eds) (1991). L'appropriation de la terre en Afrique noire : manuel d'analyse de décision et de gestion foncières. Paris : Karthala, 359 p. (Economie et Développement).

19. Le Meur, P-Y. (2008). L'information foncière, bien commun et ressource stratégique. Le cas du Bénin. Londres, IIED, Dossier n ${ }^{\circ} 147$, $28 \mathrm{p}$.

20. Léna, Ph. (1979). Transformation de l'espace rural dans le front pionnier du sud-ouest ivoirien. Abidjan : ORSTOM, $345 \mathrm{p}$.

21. Lesourd, M. (1988). La forêt, la machette et le billet de banque Problématique du colonat agricole en région d'économie de plantation : l'exemple du système de production Baoulé (Côte d'Ivoire forestière). Cah. Sci. Hum. 24 (1) pp 73-97.

22. Lesourd, M. (1982). L'émigration Baoulé vers le Sud-ouest de la Côte d'Ivoire. Thèse de $3^{\mathrm{e}}$ cycle, Université Paris Nanterre.

23. Mlan, K. S. (2015a). Emergence de l'hévéaculture, conflits et disparition de la riziculture familiale dans l'écotone (Centre de la Côte d'Ivoire). Abidjan, RSS-PASRES, pp 78-91.

24. Mlan, K. S. (2015c). «Une localité, un espace reboisé : une réponse à la concentration foncière et aux « jeux de pouvoirs » des dirigeants politiques en Côte d'Ivoire. Abidjan, Revue Africaine d'Anthropologie, Nyansa-Pô, $\mathrm{N}^{\circ} 19$, pp99-117.

25. Mlan, K. S . (2013). Le rônier, Borassus aethiopum Mart., dans la vie sociale et culturelle des Baoulé Aïtou de Dimbokro (Côte d'Ivoire). Abidjan, Thèse unique de Doctorat, Université Félix Houphouët Boigny, 445p.

26. Mlan, K. S . (2004). Processus de valorisation des écosystèmes secondaires et son impact sur les rapports sociaux : cas de Gnamagui dans la sous-préfecture de Méagui. Mémoire de Maîtrise, Unviversité de Cocody, Abidjan 
27. Peltre, P. (1977). Le "V Baoulé" (Côte d'Ivoire centrale) : héritage géomorphologique et paléoclimatique dans le tracé du contact forêtsavane. Paris, ORSTOM, (80), 198 p. (Travaux et Documents de l'ORSTOM ; 80). Th. 3e cycle Géogr.

28. Ruf, Fr. (2012). L'adoption de l'hévéa en Côte d'Ivoire. Prix, mimétisme, changement écologique et social. Economie Rurale -330331, pp 103-124

29. Ruf, Fr. (2013). Agriculture contractuelle et boom de l'hévéaculture villageoise en Côte d'Ivoire. Cahiers Agricultures, 22 (1), pp 46-52

30. Schwartz, A. (1979). Colonisation agricole spontanée et émergence de nouveaux milieux sociaux dans le Sud-Ouest ivoirien : l'exemple du canton Bakwé de la sous-préfecture de Soubré. Cahiers ORSTOM. Série Sciences Humaines (FRA), Vol. 16, N¹-2, pp 83-101

31. Stamm, V. (1998). Structures et politiques foncières en Afrique de l'Ouest. L'Harmattan, Paris.

32. Tarrouth, H. G. (2016). Les acquisitions de terres rurales par les cadres en Côte-d'Ivoire : enchâssement social, conflits, sécurisation, Thèse de Doctorat unique, Université Félix Houphouet-Boigny, IES, Cocody, Abidjan, Côte-d'Ivoire.

33. Tarrouth, H.G. \& Colin, J.-Ph. (2016). Les acquisitions de terres rurales par les "'cadres"' en Côte d'Ivoire : premiers enseignements. Cahiers Agricultures. 25 : 15005 [6p].

34. Verdeaux, F . (2011). Des forêts et des hommes : 14. Politiques et dynamiques forestières : et la déforestation ? : comment nous nous sommes disputés. (notre forêt) : politiques forestières et développement en Côte d'Ivoire. Les dossiers thématiques de l'IRD, Paris, SUDS-IRD |

35. Viti, F. (1991). Anthropologie d'un pouvoir local : histoire et politique dans l'Aitu Nvle (Baule, Côte d'Ivoire)., Paris, EHESS-ORSTOM, $671 \mathrm{p}$.

36. Zamblé Bi, Z. B. (2019). Déterminants de la réinsertion des migrants de retour dans le jeu foncier de leur zone de départ: cas de Zoukougbeu (Centre-ouest Côte d'Ivoire). Mémoire Master en sociologie, Daloa, Université Jean Lorougnon Guédé. 